\title{
The smallest bioreactor
}

A method for encapsulating single cells or single organelles in aqueous droplets combines optical trapping and microfluidic droplet generation techniques.

Move over, test tubes. Laboratories are going nanoscopic as microfluidic technologies make it increasingly possible to study biochemical reactions in ever-shrinking volumes. Toward this effort, an innovative method for the encapsulation of single cells or single organelles in picoliter to femtoliter aqueous droplets was recently introduced by Daniel Chiu and colleagues at the University of Washington.

In a recent paper from Analytical Chemistry, Chiu and colleagues describe two strategies that combine microfluidic droplet generation techniques with optical trapping to selectively and stably encapsulate a single cell or organelle. One strategy uses a T-channel design, in which the aqueous phase is introduced perpendicularly to the flowing oil phase. When pressure is applied to the aqueous phase, droplets are continuously sheared off by the oil stream. The other method involves channel constriction, in which the aqueous phase is squeezed through a narrow channel leading to a large oil reservoir. The plugs of water that form in the channel become droplets upon reaching the oil reservoir. In both techniques, optical tweezers are applied to place the cell or organelle at the oil-water interface, so that the particle is encapsulated within the forming droplet. In a notable improvement over previous microfluidics designs, "Using optical methods to move cells around gives the user excellent control and flexibility," says Chiu.

Once imprisoned within an aqueous droplet, particles with high surface charges such as cells and organelles cannot be forced back across the water-oil interface, and are thus stably confined. The successful encapsulations of a single B lymphocyte cell (Fig. 1) and a single mitochondrion were accomplished, demonstrating the potential of the method for single-cell and single-organelle studies. "Single-cell studies are interesting because there are cell-to-cell variations," explains Chiu, "and for some applications there are not that many cells to start with, for example, if you are looking at rare cells."

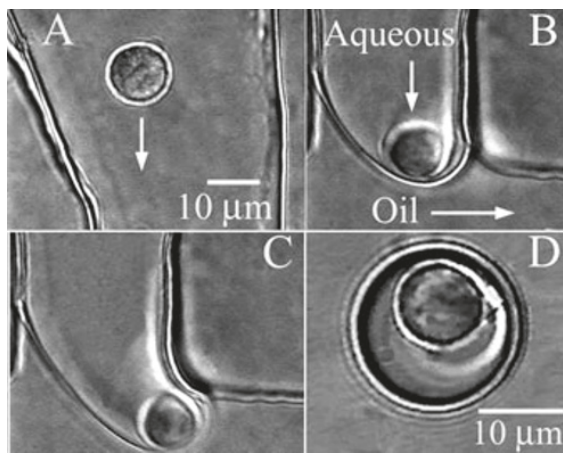

Figure 1 | Encapsulation of a single B lymphocyte in an aqueous droplet in silicone oil. (a,b) Optical trapping is used to position the cell at the wateroil interface. (c) The aqueous droplet is sheared off by the flowing oil phase. (d) Finally the cell is encapsulated in the aqueous droplet. Reprinted from Analytical Chemistry with permission from the American Chemical Society, copyright 2005.

Because the particle of interest is confined to a droplet volume not much larger than that of the particle itself, the concentration of cell contents before and after lysis remains essentially the same, which is key to be able to perform biochemical assays with single cells. To demonstrate the utility of this technique for such studies, the researchers conducted an enzymatic activity assay. A mast cell was trapped within a droplet containing fluorescein di- $\beta$-D-galactopyranoside (FDG), the fluorogenic substrate of intracellular $\beta$-galactosidase. Following photolysis and release of $\beta$-galactosidase, the droplet became highly fluorescent owing to the accumulation of the reaction product, fluorescein. Without confinement to the miniscule droplet volume, dilution would have made the visualization of fluorescein difficult to follow. "You can use very high resolution microscopy to look at cell and subcellular structures. But you can't get much biochemical information using microscopy," says Chiu. "We are trying to develop a platform by which we can do very small-scale transformations and then be able to get the kind of information that you can only get with bulk biochemical assays, but at the level of the cell or organelle."

\section{Allison Doerr}

\section{RESEARCH PAPERS}

$\mathrm{He}, \mathrm{M}$. et al. Selective encapsulation of single cells and subcellular organelles into picoliter- and femtolitervolume droplets. Anal. Chem. 77, 1539-1544 (2005) 